\title{
Early night-time-bracing - an alternative in AIS management
}

\author{
Andreas Selle*, Jens Seifert, Carl Gustav Carus \\ From 7th International Conference on Conservative Management of Spinal Deformities \\ Montreal, Canada. 20-22 May 2010
}

\section{Introduction}

As a result of physical and psychological stress we often find very weak compliance for traditional Scoliosis FullTime-Bracing (FTB) in comparison to Night-Time-Bracing (NTB) [1]. The investigation tries to find out whether or not Early Night-Time-Bracing (ENTB, 16-25 COBB) can effectively stop AIS progression and prevent Full-Time-Bracing.

\section{Material and methods}

Out of 671 AIS brace-patients we selected the ENTBpatients. Out of them we excluded patients with further diseases, patients who moved, patients with very high reaching thorathic curves and patients with follow-up's of less than 3 years. That left us with a small group of 20 patients with initial COBB angles $16-25^{\circ}$, all treated in one institution with a Dresdner NTB, without further treatment, with an average age of 11.6 years at brace discontinuation and an average follow-up of 5 years (3.1 - 7.8). Treatment was considered successful if there was improvement or at least $5^{\circ}$ progression of primary curve and no progression (neither primary nor secondary curve) above absolute value of $25^{\circ} \mathrm{COBB}$.

\section{Results}

We obtained primary correction of $87.8 \%$. After an average of 5.2 years $(3.1-7.8)$ we found "true correction" (upright, standing position without wearing the brace) of $24.4 \%$ for primary curve and $12.2 \%$ for secondary curve. All in all we obtained a success rate of $85 \%$ (improvement or constancy).

We could suspend treatment in 4 cases before Risser 4 because of single-digit COBB-angles after an average of 1.9 years $(1.1-3.4)$. We had to complete the treatment to Full-Time for 3 patients (15\%) because after longer

\footnotetext{
Orthopaedie- und Rehatechnik Dresden GmbH, Dresden, Germany
}

Full list of author information is available at the end of the article periods of NTB their COBB-angles got worse and crossed the $25^{\circ} \mathrm{COBB}$-mark. They nonetheless had comfortable Part-Time-Bracing times of an average of 4.6 years $(2.3-6.6)$. Their progression could be stopped by FTB. No one passed the $30^{\circ} \mathrm{COBB}$-mark and no surgery was needed.

\section{Discussion}

93.8\% of our patients feel their QOL has been affected by a brace [1]. $91.4 \%$ of full-time treated patients identified daytime as the most frustrating time [1]. Accordingly, ENTB of moderate curvatures (16-25 $)$ seems to be the bracing method with the lowest physical and psychological impact, combined with a low risk of overtreatment (daytime life without any restrictions, possibility to suspend the night-treatment if scoliosis decreases significantly). ENTB shows an excellent success rate (85\%). It even has an obvious option for the $15 \%$ failures (change to FTB) and is still beneficial for these patients, because it also enlarges their period of living without a brace during the daytime. AIS patients with initial COBB-angles $>25^{\circ}$ have not been included in the investigation.

\section{Conclusion}

ENTB of moderate curvatures is a sufficient method to stop AIS progression and to avoid FTB. It should become an integral part of AIS therapy in between only physiotherapy and Full-Time-Bracing.

Published: 10 September 2010

Reference

1. Andreas Selle, Jens Seifert, Carl Gustav Carus: Compliance comparison of different bracing concepts. Scoliosis 2010, 5(Suppl 1):059.

doi:10.1186/1748-7161-5-S1-O57

Cite this article as: Selle et al:: Early night-time-bracing - an alternative in AIS management. Scoliosis 2010 5(Suppl 1):057. 